\title{
Dropout in secondary education: An application of a multilevel discrete-time hazard model accounting for school changes
}

\author{
Carl Lamote . Jan Van Damme . Wim \\ Van Den Noortgate • Sara Speybroeck • \\ Tinneke Boonen • Jerissa de Bilde
}

Received: October 7, 2011 / Accepted: January 3, 2012

\begin{abstract}
For several decades, researchers have focused on dropout in search for an explanation and prevention of this phenomenon. However, past research is characterized by methodological shortcomings. Most of this research was conducted without considering the hierarchical structure of educational data and ignored the longitudinal path towards dropout. Moreover, research that did take into account these shortcomings, did not correct for student mobility between schools, despite the strong correlation with dropout (South et al. 2007). In this study, we attempt to address these shortcoming by implementing a multilevel discrete-time hazard model and exploring the effect of different school classifications on the school effects. Partially analogous to Grady and Beretvas (2010) we compare models with estimated school effects based on the first and on the last school attended and compare these models with multiple membership models and cross-classified models. The results of this comparison indicate that ignoring student mobility can have strong implications on the predictors of dropout. Not only do models which take into account this mobility yield better model fits, models ignoring this mobility tend to miss the effect of school level variables. With respect to the conclusions on dropout research, our models provide evidence for the often cited student characteristics predicting dropout and indicate stronger school effects than generally assumed.
\end{abstract}

Keywords discrete-time hazard analysis · cross-classifications · multiple membership · dropout · secondary education · methodology of longitudinal research

C. Lamote · J. Van Damme · S. Speybroeck · T. Boonen · J. de Bilde Centre for Educational Effectiveness and Evaluation,

The Education and Training Research Group,

KU Leuven, Dekenstraat 2, P.O. Box 3773, 3000 Leuven, Belgium

E-mail: carl.lamote@ppw.kuleuven.be

W. Van Den Noortgate

Methodology of Educational Sciences Research Group, K. U. Leuven,

Andreas Vesaliusstraat 2, P.O. Box 3762, 3000 Leuven, Belgium 


\section{Introduction}

For the majority of students, graduating from secondary education can be seen as obvious and unproblematic, at least in industrialized countries. However, a part of the students never reaches this endpoint and leaves secondary education before receiving a diploma. These students are termed as dropouts. Over the past 25 years, numerous studies have focused on the dropout phenomenon in secondary and also in higher education (e.g. Finn 1989; Lee and Burkam 2003; Rumberger 2001; Roderick 1994). The focus on dropout in secondary education can be explained by the severe consequences of this dropout: in an age where diplomas continue to gain in importance, leaving school without a qualification implies a strong decrease in future employment opportunities (Solga 2002). To classify the numerous variables considered in past research on dropout, Rumberger (2001) distinguished two broad perspectives to look at student dropout: an individual and an institutional perspective.

The individual perspective encompasses the majority of regularly observed explanatory variables for dropout. Several of these variables are also used in this study and include gender, (prior) achievement, grade retention, student mobility and student engagement. One of the most commonly observed student characteristics predicting dropout, is gender. Although regularly observed, the effect of gender on dropout was not always unequivocal: a first set of studies found that boys tend to have higher odds of leaving school (e.g. Alexander et al. 2001; Marks 2007), a second set concluded an opposite effect with girls having higher odds of leaving school (Croninger and Lee 2001; Goldschmidt and Wang 1999) and a third set of studies found no effect of gender at all (Lee and Burkham 2003; Hickman et al. 2008). The strong relation between dropout and grade retention in primary and secondary school received also a lot of attention (e.g. Alexander et al. 2001). In their review, Jimerson et al. (2002) pointed on the strong predictive power of (early) grade retention on dropout. For example: Rumberger (1995) concluded that students who repeated a grade, were nearly 11 times more likely to drop out compared to students who had not repeated any grade. Besides gender and grade retention, low-achievers are often at higher risk for dropout. Alexander et al. (2001) concluded that low achievement in first grade, was one of the major predictors of dropping out. Another relevant predictor of future dropout, is student mobility. Students who experienced one or more school changes, are approximately twice as likely to drop out compared to non-mobile students (South et al. 2007). Dropping out of secondary education is not only a matter of gender, grade retention, or low school performance, but can be an expression of a more subtle and underlying process of reduced school engagement. School engagement was included in many dropout theories, but not often considered in empirical research (see e.g. Ream and Rumberger 2008). However, Finn's (1989) identification-participation model stated that active participation in school and a feeling of identification with school, strongly mediates school outcomes.

In addition to the individual perspective, also the institutional setting in which students live can predict future dropout. Regarding this institutional perspective, we consider the family background of the student and the schools attended. The family background is usually operationalized by the socioeconomic status (SES) of the student. As for SES, especially the educational level of (one of) the parents and their income remain an important contributor to success in school. Even 
when controlling for other student characteristics, students with a low SES background have a higher dropout probability (Alexander et al. 2001). Concerning the second institutional variable, research is much more limited as only a few studies included school characteristics in a multilevel model, which led to several new findings (Goldschmidt and Wang 1999; Lee and Burkam 2003; Luyten et al. 2003; Rumberger 1995). Lee and Burkam (2003) focused on school academic and social organization and concluded that students were less likely to drop out in schools where relationships between teachers and students are more positive. Furthermore, Rumberger and Thomas (2000) concluded that high-SES schools had $40 \%$ lower dropout rates than average SES schools, whereas low-SES schools had $60 \%$ higher dropout rates than an average SES school.

Rumberger (2001) stated that dropout is a final action in a long-term process. Nevertheless, several of the mentioned studies have a narrow focus on the dropout phenomenon itself (e.g. Luyten et al. 2003). Indeed, research should focus on the longitudinal nature of dropout, instead of drawing conclusions based on one moment, since not every predictor seems to have the same effect at every moment. Only few studies took this longitudinal nature into account. Bowers (2010) concluded that time-varying variables in a longitudinal study (e.g., grade retention, achievement scores) predicted dropout more than time-invariant variables (e.g., gender).

Besides ignoring the longitudinal nature of the data, research also often ignores several aspects at the institutional or school level. Such school characteristics, as well as student characteristics, can be integrated into a hierarchical model, with students nested in schools. As mentioned earlier, this multilevel approach was rarely used. To account for both the longitudinal and hierarchical structure of the data, we propose using a longitudinal multilevel model. A complicating factor however, is that a pure nesting of students within schools is not always in accordance with educational reality, where students encounter school changes during their career through (secondary) education. When focusing on school effects, changing schools entails a change of school environment and thereby other values for the school level variables. Students who attended multiple secondary schools, have been exposed to multiple school effects (Grady and Beretvas 2010), and most of the time these school changers are no random group and should therefore not be excluded from a dataset (Goldstein 1997). As mentioned earlier, drop-out was found to be related with student mobility, pointing to the importance of including mobile students in a study on dropout. This change extends the usual nested structure of educational data, and suggests the use of cross-classifications and multiple membership models (Goldstein 2011).

\section{Research Questions}

Taking into account the aforementioned methodological considerations, and the theoretical background of dropout in secondary education, we propose four research questions. The first research question is the main interest of this study and concerns the methodological approach in studying dropout, where we are interested in the most appropriate model for modelling dropout in secondary education. More specifically, the first research question deals with the longitudinal character of the data and the multiple schools attended. We will compare a model with 
a multiple membership structure, a model with a cross-classification and models only focusing on one school. We expect to find a better model fit when taking all the schools attended into account. We will also compare the methods regarding the answers they give on the three following substantive research questions. In the second research question, the effect of student characteristics on the chance to dropout is addressed. In line with past research on dropout, we expect a higher chance to dropout with male students, who repeated a grade in primary and/or secondary education and grew up in a low SES family. The third research question addresses the effect of school level variables on the chance to dropout. Although previous research rarely focused on school level variables, the research that did, found, among others, effects of school composition, school size and relationships. The fourth research question follows directly from the longitudinal approach of modelling dropout in this study and will identify the more hazardous grades for dropout in secondary education.

The choice of the variables on the student level and school level is mainly based on the research literature on dropout, and therefore the study will partially replicate previous studies. The main contribution of this study is, as mentioned, the implementation of alternative models accounting for the longitudinal character, the multilevel structure and the school changes. The next part presents the dataset, which we used to test the proposed models and from which we will draw our overall conclusions. The description of the dataset is followed by a short introduction into hazard modelling, multiple membership and cross-classified models in the method section. We end the method section with an integration of these multiple membership and cross-classified models into a hazard analysis.

\section{Data source}

The data were drawn from the Flemish 'LOSO'-project (Van Damme et al. 2002). This longitudinal research project started in 1990 and followed a cohort of 6411 students in 57 secondary schools. These students were followed through secondary education, with a follow-up through higher education or the first years on the labour market. The LOSO-dataset contains data at the student, class, teacher and school level. In this study, we selected a subsample of 4735 pupils in 55 secondary schools, of which 514 dropouts, who started in 1990 in the first year of (regular) secondary education (grade 7) and had known educational positions and school ID's for every year through secondary education. Students who repeated a grade and/or changed schools were also included. Because of our interest in the effect of school characteristics on dropout rates, and because we have information on school characteristics for LOSO-schools only, we excluded students transferring to a school that was not included in the LOSO-project. The LOSO-project selected secondary schools from several regions in the Flemish community, and in the selected regions, almost every school participated. Because of the strong coverage within every region, we were able to follow-up the students who changed schools within the region and we can assign school characteristics to every student for every time point. 


\subsection{Measures}

A student is considered as dropped out at the moment (s)he leaves fulltime secondary education, and is not able anymore to obtain a diploma of secondary education (a full high school diploma). This definition of dropout is very strict and as a result, students who leave fulltime secondary education for part-time education or for other alternative schoolings are considered as dropouts although in the Flemish community they still can receive a qualification. This qualification however is not comparable to a diploma of secondary education, since such qualification does not guarantee the same chances in higher education and on the labour market.

We selected five student background characteristics that will be included in our models as independent variables: gender (GEN), initial cognitive ability (COGN), socioeconomic status (SES), older at start of secondary education than the norm student (RET_P) and retained in grade during secondary education (RET_S). Gender (GEN) is implemented as dummy variable, with value ' 1 ' for girls, and boys with value ' 0 ' as reference category. The initial cognitive ability (COGN) includes three components: math achievement, language achievement and intelligence, measured at the start of grade 7 . All the components had high internal consistencies, ranging between $\alpha=.82$ and $\alpha=.93$ (Van Damme et al. 1997).

The math achievement test was a multiple-choice test which consisted of 50 items, assessing set theory, algebraic problems and geometry. The raw scores were converted into item response theory (IRT) scores. The content was in line with the content received in the highest grades of primary school. The language achievement test consisted of 100 items, divided into 4 sections: spelling, grammar, language use and comprehension. The intelligence score was based on the Getlov-intelligence test, which measured the intelligence of students at the beginning of secondary education. This test comprises three test scores: a score for verbal intelligence, a score for spatial representations, and a score for numerical intelligence. A factor analysis on the 5 intelligence and achievement scores, gave a one factor solution. The initial cognitive ability predictor we will use (COGN) are factor scores resulting from this factor analysis. Students with missing values on one or more of the 5 scores were excluded from further analysis. The socioeconomic status (SES) is based on parental education, parental occupational status, monthly income and a variable measuring the cultural capital of the family. These variables were combined into a factor analysis, resulting in a one factor solution. Because we only have selfreported information on grade retention in primary education, we deduct whether or not the student was retained in grade from his age (RET_P). Traditionally, students start in secondary education in the year they reach the age of 12 years. Students who were older at the start of secondary education, most likely repeated a grade in primary education and are coded ' 1 '. Students who started secondary education at the age of 12 (or younger), are coded ' 0 '. Grade retention in secondary education (RET_S) is implemented as a time-varying dummy variable, with value ' 1 ' in the grade in secondary education which the student repeated and in the subsequent grades.

Besides the student background characteristics, we included the following school composition variables: socioeconomic school composition (SES-S), cognitive composition of the school (COGN-S) and gender composition of the school (GEN-S). These variables are aggregations of the student-level variables SES, COGN and GEN. Although we only incorporate students belonging to the LOSO-cohort in 
this study, the construction of these aggregated variables is based on all the students for which this information was available, including non-LOSO students, as recommended by Snijders and Bosker (1999).

An indicator of the school climate, referring to the relationship with teaching staff and peers, reported by the students (RELAT-S) was also included. As Fredricks et al. (2004) mentioned, the relationship between student and teachers on the one hand, and the relationship between student and peers on the other, is a strong indicator of the emotional engagement towards the school. Therefore, we implemented this RELAT-S. This variable is part of a larger set of school climate variables, constructed by De Fraine (2003), and was also implemented in Opdenakker and Van Damme (2005). RELAT-S consists of three first order variables: (1) relationship with teachers, with items as 'Teachers trust the students' and 'Teachers give personal attention to every student'; (2) granting equal rights to every student, with items as 'Teachers behave differently depending on the track' and (3) friendships amongst students, with items as 'New students are accepted quickly'. Because of the strong correlation between these three variables, they were combined into a new, second order variable relationships at school.

\section{Method}

In this study, we are not only interested in 'whether' students drop out of secondary education, but also in 'when' students drop out. As Singer and Willett (2003) suggested, this question is answered by making use of a discrete-time hazard analysis (also known as discrete-time event-history analysis or discrete-time survival analysis). However, making use of standard discrete-time hazard techniques would ignore the fact that students are clustered in schools and tend to change schools during secondary education. In the next sections, we discuss the multilevel discrete-time hazard (MDTH) approach and extend this approach by taking into account student mobility, making use of a multiple membership model and models with cross-classifications. First, we discuss the benefits of this MDTH approach compared to the more 'traditional' approach in studying dropout, followed by a short introduction to multiple membership models and models with cross-classifications. We end this method section with the integration of the multiple membership structure and the cross-classifications into the MDTH model.

\subsection{Multilevel discrete-time hazard model}

In this study, we opt for a multilevel discrete-time hazard analysis (MDTH) for four main reasons: the interest in predicting the (non) occurrence of an event (here: dropout), the longitudinal character of our data, the problem of tied observations and the hierarchical structure of educational data.

Hazard analysis is a flexible (and straightforward to implement) tool which measures the (non) occurrence of an event, as well as the timing of the event. This time-to-event analysis starts at a well-defined starting point (here: at the beginning of grade 7) and ends at the occurrence of the targeted event (here: dropout), predicting the event-occurrence at each time-interval. However, because of the longitudinal character of our data we may encounter a common aspect of 
longitudinal data analysis: censoring. Censoring occurs when some participants do not experience the studied event during the time period of the study. In educational context, when studying dropout, this censoring might occur in different manners: students did not experience the event when the study ended, students were lost to follow-up or students withdrew from the study because of another event not of interest (e.g. death) (Kleinbaum and Klein 2005). In our study, censoring can only occur at Grade 12, when students did not experience the targeted event and stayed in the dataset until graduation. For the hazard analysis in this study, a discrete-time approach seems the most appropriate method, since our data encloses multiple grades. Most of the individuals have a dropout date that matches the end of a school-year, or the beginning of a subsequent school-year, which raises the problem of tied observations (two or more students experience the event at the same time). Such tied observations are not uncommon in educational research, where events are often observed or take place at the end of a school-year. Using a continuous-time approach despite tied observations can lead to biased estimates (Allison 1982). A possible and easy to implement solution is to treat time as if it were discrete. Although not every student drops out at the last day of the school-year, this discrete-time approach seems a good approximation of the moment of dropout. The discrete-time hazard analysis allows us to use the logistic regression model, with the addition of time-dummy variables for every grade and time-invariant and/or time-varying covariates. Traditional discrete-time hazard analysis assumes that students behave independently from each other. This is not the case in an educational context, where students are grouped in classes and schools, and observations of students within the same school are not independent from each other. Therefore, a multilevel structure with individuals at Level-1, grouped into Level-2 units (schools) is more appropriate. As such, the multilevel discrete-time hazard model is written as (Goldstein 2011; Barber et al. 2000):

$$
\log \left(h_{t i j} /\left(1-h_{t i j}\right)\right)=\beta_{0}+\alpha_{t}+\beta x_{t i j}+u_{j}
$$

where $h_{t i j}=\operatorname{Pr}\left(Y_{t i j=1} \mid Y_{(t-1, i j)}=0\right)$ is the hazard that student $i$ in school $j$ drops out at time $t$ (given the event has not yet occurred to that individual before time $t), \alpha_{t}$ is a function of time, $\beta$ a vector of parameters representing effects of covariates $x$ on the probability of event occurrence and $u_{j}$ the school-level random effect.

In a traditional multilevel discrete-time hazard model, students are clustered in only one school and consequently, researchers need to allocate only one school ID for every pupil. This allocation is straightforward for students attending only one school during the study, but becomes problematic for students changing schools. For these school changers, several ad-hoc approaches for the choice of school ID's have been adopted in previous research. One of the possible solutions is to take the school ID of the last or the first school attended. Using the school ID (and corresponding school characteristics) of the first or last school, however, substantially underestimates the true between-school variance (Goldstein 2011; Goldstein et al. 2007; Grady and Beretvas 2010). Another solution for this school change is to simply delete the mobile students. The resulting reduced dataset, however, may be biased as most of the time, this group of school changers is no random group (Goldstein 1997). The following sections go more deeply into the matter of modelling student mobility, by making use of multiple membership models and 
cross-classified models. The resulting data structure is not strictly hierarchical anymore, but non-hierarchical in which repeated measures are cross-classified by students and schools. Although the benefits of these approaches are widely studied (see e.g. Grady and Beretvas 2010; Luo and Kwok 2009; Fielding 2002), only few educational studies took this non-hierarchical structure into account (e.g. Goldstein and Sammons 1997; Pustjens et al. 2008; Raudenbush 1993; Teitler and Weiss 2000). In this study, we give a short introduction into these approaches, implement these into a discrete-time hazard analysis and present an application of these approaches.

\subsection{Multiple membership models}

Because many students change school at least one time, we could incorporate this school movement by making use of a non-hierarchical approach where the lower level units (here: students) are members of more than one higher level unit, resulting in a multiple membership model.

Because we have a school ID for every school in every grade, and we know the grade of dropout, we are able to assign weights to every secondary school the student attended. By assigning weights proportionally to the time spent in every school, we implicitly assume that each year the school has the same importance. One could argue that the last school attended has a more substantial impact compared to the first school attended, and should therefore receive a higher weight, irrespective of the time spent in the last school. On the other hand, Browne et al. (2001) and Goldstein et al. (2007) experimented with different weighting schemes, and concluded that the parameter estimates are (relatively) insensitive to the choice of weighting scheme.

Taking into account this multiple-membership, the basic hierarchical logistic model is extended to a model with a multiple membership structure (Goldstein 2011):

$$
\begin{array}{r}
\operatorname{logit}\left(\pi_{i j}\right)=\beta_{0}+\sum_{j \in \operatorname{school}(i)} w_{(i, j)} u_{j} \\
\text { withu }_{j} N\left(0, \sigma_{u}^{2}\right) \text { and } \sum_{j \in \operatorname{school}(i)} w_{(i, j)}=1
\end{array}
$$

In Equation $(2), w_{(i, j)}$ is the weight assigned to the random effect for student $i$ in school $j$. For each student, the sum of these weights equals 1 . As a result of these weights, we must be careful when interpreting the variance components of the effects. As Leckie (2009) states, the variance $\sigma_{u}^{2}$ of the school effect varies along with the mobility of the student. A student who attended only one school, has a contribution of the school to the variance of the student of $\sigma_{u}^{2}$, but students who experienced school change will have a contribution of the school to the variance of the student, proportional to the time spent in a particular school. Consider for example a pupil who spends 4 years in school $\mathrm{A}$ and 2 years in school B, the model is as follows (Goldstein 2011):

$$
\begin{gathered}
\operatorname{logit}\left(\pi_{i j}\right)=\beta_{0}+w_{1 i, j A} u_{j A}+w_{2 i, j B} u_{j B} \\
\text { with } \mathrm{w}_{1 i, j A}=4 / 6 \text { and } w_{2 i, j B}=2 / 6
\end{gathered}
$$


and in calculating the total contribution of the attended schools, the weighted effects of every school attended are summed to:

$$
\left.\sigma_{u}^{2}=w_{(} 1 i, j A\right)^{2} \sigma_{u} A^{2}+w_{(} 2 i, j B^{2} \sigma_{u} B^{2}
$$

This is the strength of these multiple membership models: the longer a student spends in one school, the greater the impact of that particular school in the final school effect. When these models are extended with variables on the school-level, this multiple membership must also be taken into account. However, computing school-effects with a multiple membership is, with the current software limitations, not obvious and time-consuming. One has to weigh each variable on the school-level with the corresponding weightings for the attended schools, for a particular student. After this weighing, the variable on the school level equals the sum of the weighted school-characteristics for every school attended. For example: a student who spent 2 year in a school with $90 \%$ girls and 4 years in a school with $69 \%$ girls will have an outcome on 'school composition by gender' of $(2 / 6)(0.9)+(4 / 6)(0.69)=0.76($ De Fraine 2003)

\subsection{Cross-classification models}

A drawback of the multiple memberships are the practical difficulties. Moreover, multiple membership models give information on the time spent in a particular school, but do not give any information on the moment the student was in a particular school. To model these school changes, we can also use a cross-classified model, where the school attended is not a fixed feature of the student, but depends on the grade attended by that student. This means a reformulation of the traditional multilevel discrete-time hazard model with students at Level-1 and schools at Level-2 to a model with observations (Level-1) nested in a combination of students and schools at Level-2. This way, schools are not characteristics of the students at Level-2, but are a characteristic of the Level-1 unit defined as the measurement moment for a student. Such a formulation reflects the model described by Raudenbush and Bryk (2002) and Fielding and Goldstein (2006) in which repeated measures are cross-classified by students and schools. With this cross-classification, there is no need to weigh every school characteristic because a difference or change in school characteristic due to school change is directly captured. The basic logistic cross-classification model is written as (Goldstein 2011):

$$
\begin{array}{r}
\operatorname{logit}\left(\pi_{i j}\right)=\beta_{0}+u_{1 i}+u_{2 j} \\
\mathrm{u}_{1 i} N\left(0, \sigma_{u_{1}}^{2}\right) \text { and } u_{2 j} N\left(0, \sigma_{u_{2}}^{2}\right)
\end{array}
$$

Equation (5) is the basic cross-classified model with $\sigma_{u_{1}}^{2}$ as the variance between students and $\sigma_{u_{2}}^{2}$ the variance between schools.

\subsection{Multiple Membership and Cross-classified Discrete-time Hazard Model}

The aforementioned methodological approaches provide a strong framework for analysing dropout in secondary education. As mentioned, changing schools is a strong predictor of this dropout and should be implemented in a model which 
makes use of the hazard approach to model dropout. When we incorporate the multiple membership in the discrete-time hazard model, the model will be:

$$
\log \left(h_{t i j} /\left(1-h_{t i j}\right)\right)=\beta_{0}+\alpha_{t}+\beta x_{t i j}+\sum_{j \in \operatorname{school}(i)} w_{i, j} u_{j}
$$

with $\alpha_{t}$ as a function of time, $\beta x_{t i j}$ as a vector of parameters representing effects of covariates on the probability of event occurrence and with the multiple membership part, represented by summation of the weighted school characteristics. When we incorporate the cross-classification in the discrete-time hazard model, the model will be:

$$
\log \left(h_{t i j} /\left(1-h_{t i j}\right)\right)=\beta_{0}+\alpha_{t}+\beta x_{t i j}+u_{j}
$$

Indeed, this cross-classified model is written in the same way as the traditional MDTH with only a random school effect and no random student effect, because a student can drop out only once and variance between students is already accounted for by the first level Bernouilli distribution. The cross-classified aspect is in the handling of the nesting of students within schools. In the traditional MDTH, a student is nested in only one school, where in this cross-classified approach, a measurement is nested within a crossing of a student and a school, and as such this school indicator and corresponding school characteristics can change over time. The latter is the main raison for adopting a cross-classification; the school characteristics are handled in a similar way as in a 'traditional' cross-classification. We will estimate different models, each model with a different approach for the school ID, partially analogous to Grady and Beretvas (2010). The different approaches are respectively the first school approach (by taking the school ID of the first school attended), the last school approach (by taking the school ID of the last school attended), the delete approach (by deleting mobile students), the multiple membership and cross-classified approach. We choose these different approaches because of the frequent implementation in previous research and to illustrate the possible bias of these approaches. These models are preceded by a model without taking into account the school level. All the models were estimated with the MLwiN software (Rasbash et al., 2009), with the Markov Chain Monte Carlo estimation. This MCMC-approach can easily handle the multiple membership and cross-classification. In order to compare model fits, we use this MCMC-approach also for the preceding models. The evaluation of model convergence was based on the trajectories of the estimates, the autocorrelation function and the RafteryLewis diagnostic as a diagnostic for the required length of the Markov chain. We compare the different model fits by making use of the deviance information criterion (DIC), where models with lower DIC values are preferred. Differences around the value of 10 or higher, are seen as substantial (Spiegelhalter et al. 2002). In the next part, we will present different results, arising from different modelling approaches. 
Table 1 Life Table

\begin{tabular}{cllccc}
\hline \hline Grade & $\begin{array}{l}\text { Enrolled at be- } \\
\text { ginning of year }\end{array}$ & $\begin{array}{l}\text { Not in subse- } \\
\text { quent year }\end{array}$ & Censored & Hazard $h$ & Survival $s$ \\
\hline 0 & 0 & 0 & & 0 & 1 \\
7 & 4735 & 2 & & 0.0004 & 0.9996 \\
8 & 4733 & 63 & 0.0133 & 0.9863 \\
9 & 4670 & 111 & 0.0238 & 0.9628 \\
10 & 4559 & 135 & & 0.0296 & 0.9343 \\
11 & 4424 & 143 & 4222 & 0.0323 & 0.9041 \\
12 & 4281 & 59 & 0.0138 & 0.8917 \\
\hline
\end{tabular}

\section{Results}

\subsection{Hazard analysis of dropout}

As one of the main features of hazard analysis, we first present a table of the number of students who drop out from the beginning of secondary education, up until the end of secondary education (Table 1), a table that in hazard analysis is called a life table. The columns of hazard and survival are essential for hazard analysis. This hazard $h$ is the conditional probability that an individual $i$ will experience the event of interest in a time period $t$, given that they did not experience the event in an earlier time period (Singer and Willett 2003). In this study, the hazard probability is a representation of the proportion of dropouts in the group of students in a certain grade which are still at risk for dropout. The interpretation of this probability is straightforward: the higher the hazard probability in a particular grade, the higher the risk for dropout in that grade. The survival estimate on the other hand, does not represent a probability in a certain grade but represents the probability that a student will 'survive' (will not experience the event of dropout) until a certain point in time and is an accumulation of the preceding hazard estimates.

A closer inspection of these hazard and survival estimates gives information on the patterns of dropout in the different grades, controlling for students who already left secondary education. At grade 7 this hazard for dropout is rather low, but gradually, this hazard increases in grade 8 until grade 10 with a peak at grade 11 and a decline in grade 12 . The survival probability indicates that $89 \%$ of the students in this dataset did not experience the event and graduate by the end of grade 12 .

\subsection{School change prior to dropout}

Changing schools during secondary education is substantial in Flanders. More than one quarter of the students in our dataset changes school once or more. This school change takes on an even larger proportion with students who drop out. Half of these dropouts changes school at least one time. An overview of school changes distributed over graduates and dropouts can be found in Table 2.

In the following paragraphs, we present the results on the research questions. At first, we compare different baseline hazard models. These baseline hazard models were estimated by making use of the first school approach, last school approach, 
Table 2 Number of school changes

\begin{tabular}{ccccc}
\hline \hline Number of school changes & \multicolumn{2}{c}{ Graduate } & \multicolumn{2}{c}{ Dropout } \\
\hline Never & 3167 & $75 \%$ & 259 & $50.40 \%$ \\
1 time & 943 & $22.40 \%$ & 206 & $40.10 \%$ \\
2 times & 100 & $2.40 \%$ & 45 & $8.70 \%$ \\
3 times & 11 & $0.20 \%$ & 4 & $0.80 \%$ \\
Total & 4221 & $100 \%$ & 514 & $100 \%$ \\
\hline
\end{tabular}

delete approach, multiple membership approach and cross-classification approach. We compare these baseline hazard models on their DIC-values.

\subsection{Baseline hazard models}

In Table 3, the first set of models are baseline hazard models, with only timevariables (Grade) as predictor for dropout. These time-variables tend toward an increase per grade until grade 11 in the risk for dropping out, with a decrease in 12 th grade. In the first model, we ignored the fact that students were nested within schools and thereby (partially) replicated past research on dropout in secondary education (see e.g. Bowers 2010; Roderick 1994). From the second model on, we adjusted for the fact that every student is in a certain school for a certain time. As such, the second model follows the traditional first school approach, where random effects are based on the first school the student attended. The DIC-values indicated an improvement of the model (Model $2 \mathrm{DIC}=4572.02$ ), compared to the first model (Model 1 DIC=4932.38), suggesting that there is variation between schools in the dropout-rate. In the third model, we took the last school attended as school classification. Comparing this with the second model, this last-school approach gives a better model fit (Model $3 \mathrm{DIC}=4422.35$ ), favouring this nesting above the first school attended. In the fourth model, we explicitly modelled school change by making use of a multiple membership structure. Less expected on the basis of the literature, this multiple membership model returned a higher DIC-value, indicating a poorer fit (Model $4 \mathrm{DIC}=4558.50$ ). There are two possible explanations: first, only the last school attended matters. Secondly, not every school attended has the same impact on dropout, and the last schools have a greater impact compared to previous schools. This brings us to the discussion by Goldstein et al. (2007) of the choice of weighting scheme. Instead of modelling this multiple membership model with different weighting schemes, we changed over to a model where time is nested within a cross-classification of a student in a certain school. Based on the DIC-value, we prefer the model with cross-classifications above the first school or last school approach (Model 5 DIC $=4375.43$ ). In the last model in Table 3 we simply deleted students who changed schools. Because this deletion resulted in a reduction of $30 \%$ of the original dataset and nearly $50 \%$ of the dropouts, we cannot reliably compare the DIC-value of the delete approach with the previous models. 
Table 3 Baseline Hazard models

\begin{tabular}{|c|c|c|c|c|c|c|c|c|c|c|c|c|}
\hline & \multicolumn{2}{|c|}{$\begin{array}{c}\text { Model } 1 \\
\text { Without school level }\end{array}$} & \multicolumn{2}{|c|}{$\begin{array}{c}\text { Model } 2 \\
\text { First school }\end{array}$} & \multicolumn{2}{|c|}{$\begin{array}{c}\text { Model } 3 \\
\text { Last school }\end{array}$} & \multicolumn{2}{|c|}{$\begin{array}{c}\text { Model } 4 \\
\text { Multiple Membership }\end{array}$} & \multicolumn{2}{|c|}{$\begin{array}{c}\text { Model } 5 \\
\text { Cross-classified }\end{array}$} & \multicolumn{2}{|c|}{$\begin{array}{c}\text { Model } 6 \\
\text { Delete approach }\end{array}$} \\
\hline & Est. & S.E. & Est. & S.E. & Est. & S.E. & Est. & S.E. & Est. & S.E. & Est. & S.E. \\
\hline Constant & $\begin{array}{l}-7.58 \\
\end{array}$ & 0.619 & -7.779 & 0.648 & -8.183 & 0.693 & -7.921 & 0.603 & -8.023 & 0.669 & -9.234 & 1.209 \\
\hline Grade 8 & $3.221^{* * *}$ & 0.632 & $3.218^{* * *}$ & 0.64 & $3.248^{* * *}$ & 0.665 & $3.160^{* * *}$ & 0.593 & $3.160^{* * *}$ & 0.64 & $4.362^{* * *}$ & 1.191 \\
\hline Grade 9 & $3.796^{* * *}$ & 0.627 & $3.823^{* * *}$ & 0.634 & $3.853^{* * *}$ & 0.659 & $3.762^{* * *}$ & 0.587 & $3.668^{* * *}$ & 0.634 & $4.632^{* * *}$ & 1.19 \\
\hline Grade 10 & $4.031^{* * *}$ & 0.625 & $4.118^{* * *}$ & 0.633 & $4.153^{* * *}$ & 0.658 & $4.050^{* * *}$ & 0.586 & $3.936^{* * *}$ & 0.633 & $4.937^{* * *}$ & 1.188 \\
\hline Grade 11 & $4.124^{* * *}$ & 0.625 & $4.225^{* * *}$ & 0.633 & $4.291^{* * *}$ & 0.658 & $4.195^{* * *}$ & 0.585 & $4.058^{* * *}$ & 0.633 & $5.022^{* * *}$ & 1.188 \\
\hline Grade 12 & $3.312^{* * *}$ & 0.633 & $3.446^{* * *}$ & 0.64 & $3.508^{* * *}$ & 0.665 & $3.401^{* * *}$ & 0.594 & $3.257^{* * *}$ & 0.641 & $4.063^{* * *}$ & 1.198 \\
\hline Between schools & & & 1.109 & 0.29 & 2.199 & 0.663 & 1.334 & 0.363 & 2.263 & 0.637 & 2.5 & 0.793 \\
\hline DIC & 493 & & 4572 . & & 4422 & & & & 4375 . & & 2370 & \\
\hline $\mathrm{N}$ & & & 2858 & & 2858 & & & & 2858 & & 2045 & \\
\hline
\end{tabular}


When we focus on the fixed effects parameter estimates, some differences arise. Compared to using a cross-classified model, ignoring the nested structure of students within schools, results in larger effects of the time-variables on dropout. This overestimation is reduced by incorporating a school-level which partitions a proportion of the variability to the school level. The variance estimates of the random effects differ much stronger. The first school approach (Model 2) resulted in a much smaller school level variance (Model $2 \sigma_{u}^{2}=1.109$ ) compared to the approaches explicitly modelling the mobility. Following Goldstein (2011), the small(er) school effect in the first-school approach could be expected because almost $28 \%$ of the pupils changed school at least one time: the limited effect of the first school on these mobile students results in a decrease of the average between school variance. When we ignore this mobility, as is the case in the first school approach, we assume that the first school attended contributes to the same extent for mobile students as for non-mobile students, which is clearly not the case. The last school approach (Model 3) yields a higher school level variance (Model $3 \sigma_{u}^{2}=2.199$ ) compared to the multiple membership model (Model $4 \sigma_{u}^{2}=1.334$ ), but a lower school effect than the cross-classified approach (Model $5 \sigma_{u}^{2}=2.263$ ). A possible explanation for these differences lies in the moment of school change or school attendance. It seems the case that not only the time spent in a particular school is of importance, but also the timing and order the student was in different schools. If we simply ignore the mobility by deleting mobile students, we get an estimate of the variance between schools that is larger than for all other models (Model $\left.6 \sigma_{u}^{2}=2.500\right)$. A closer inspection of our data gives a possible explanation for this large increase in school level variance. Comparing the dataset of the delete approach with the dataset of the first school or last school approach, one can see a large reduction in number of students and number of dropouts, which has a greater impact in schools with a significant mobility. In the first school approach, e.g. school ID 66628 has 13 dropouts on a total of 117 students; deleting the mobile students in this school, reduces the number of dropouts to 0 on a total of 58 remaining students. Deleting the mobile students has some consequences for the overall dataset: schools with a high (outgoing) student mobility, tend to have fewer dropouts, since dropout is related to changing schools. Therefore, differences in dropouts between schools become greater, resulting in an increase in school-level variance. Because we explicitly want to account for mobile students in our dropout study, we drop this approach from further analysis. The last school approach and the cross-classified approach outperform the first school, the multiple membership approach and delete approach. Therefore, we will base our further comparisons of hierarchical and non-hierarchical models only on the last school and cross-classified approach. Concerning the research questions on dropout, we add student and school characteristics to the cross-classified baseline model, because this model yielded the best DIC-value.

\subsection{Student and school characteristics}

Starting from model 7 until model 9, we add student characteristics to the crossclassified model and evaluate the model fits. To facilitate the interpretation of the continuous variables, these variables were centred around their grand mean value. The effect of the predictor variables on the odds to dropout, is expressed as the 
Table 4 cross-classified models with student and school characteristics

\begin{tabular}{|c|c|c|c|c|c|c|c|c|}
\hline & \multicolumn{2}{|c|}{ Model 7} & \multicolumn{2}{|c|}{ Model 8} & \multicolumn{2}{|c|}{ Model 9} & \multicolumn{2}{|c|}{ Model 10} \\
\hline & Est. & S.E. & Est. & S.E. & Est. & S.E. & Est. & S.E. \\
\hline Constant & -8.529 & 0.924 & -8.532 & 0.623 & -9.292 & 0.813 & -9.309 & 0.64 \\
\hline Grade 8 & $3.181^{* * *}$ & 0.905 & $3.127^{* * *}$ & 0.625 & $3.574 * * *$ & 0.787 & $3.110^{* * *}$ & 0.631 \\
\hline Grade 9 & $3.596^{* * *}$ & 0.899 & $3.637^{* * *}$ & 0.619 & $4.075^{* * *}$ & 0.788 & $3.600 * * *$ & 0.626 \\
\hline Grade 10 & $3.857 * * *$ & 0.898 & $3.950 * * *$ & 0.617 & $4.403^{* * *}$ & 0.79 & $3.912^{* * *}$ & 0.624 \\
\hline Grade 11 & $3.978^{* * *}$ & 0.898 & $4.114^{* * *}$ & 0.618 & $4.574^{* * *}$ & 0.792 & $4.078^{* * *}$ & 0.624 \\
\hline Grade 12 & $3.228^{* * *}$ & 0.905 & $3.349 * * *$ & 0.628 & $3.816^{* * *}$ & 0.795 & $3.317^{* * *}$ & 0.632 \\
\hline $\mathrm{RET}_{S}$ & $0.977^{* * *}$ & 0.099 & $0.907 * * *$ & 0.099 & $0.916^{* * *}$ & 0.103 & $1.418^{* * *}$ & 0.155 \\
\hline $\operatorname{RET}_{P}$ & $1.395^{* * *}$ & 0.095 & $0.978 * * *$ & 0.103 & $0.987 * * *$ & 0.112 & $1.766^{* * *}$ & 0.148 \\
\hline GEN & & & $-0.536 * * *$ & 0.111 & $-0.485 * * *$ & 0.114 & $-0.440 * * *$ & 0.11 \\
\hline COGN & & & $-0.720 * * *$ & 0.082 & $-0.692^{* * *}$ & 0.087 & $-0.669 * * *$ & 0.08 \\
\hline SES & & & $-0.297 * * *$ & 0.034 & $-0.276 * * *$ & 0.037 & $-0.670 * * *$ & 0.068 \\
\hline SES-S & & & & & $-1.394 * * *$ & 0.289 & $-1.269 * * *$ & 0.283 \\
\hline RELAT-S & & & & & $-1.553^{* *}$ & 0.629 & $-1.419^{*}$ & 0.604 \\
\hline $\mathrm{RET}_{P} * S E S$ & & & & & & & $0.494^{* * *}$ & 0.068 \\
\hline $\mathrm{RET}_{S} * S E S$ & & & & & & & $0.325^{* * *}$ & 0.071 \\
\hline Between schools & 1.245 & 0.399 & 0.369 & 0.144 & 0.166 & 0.076 & 0.143 & 0.065 \\
\hline DIC & 4118. & & 3967. & & 3929. & & 3881 & \\
\hline $\mathrm{N}$ & 285 & & 285 & & 285 & & 285 & \\
\hline
\end{tabular}

percentage increase or decrease in the odds due to a one-unit increase, i.e. a one standard deviation increase in the predictor variable (Pampel 2000).

At first, we add two variables referring to grade retention in the past school career, without controlling for other background characteristics. These two variables, RET_P $\left(p_{i} .001\right)$ and RET_S $\left(p_{i} .001\right)$ both have a significant effect on later dropout. This age of entry in secondary school seems to have a very strong impact on the chance for dropout: with an odds-ratio of 4.03, the odds of leaving secondary education without diploma for students who were older at the start, were about four times higher compared to students with the common age of entry. Also grade retention in secondary education (independent of grade retention in primary education) is a strong predictor of future dropout. The effect of grade retention on dropout is shown in the odds-ratio of 2.67 , indicating that for students who repeated a grade, the odds of dropping out are $165 \%$ higher compared to students who never repeated a grade. Inclusion of the remaining student characteristics, GEN, COGN and SES, further lowers the DIC-value, indicating a better model fit $(\mathrm{DIC}=3967.253)$. Adding these covariates lowers the odds of age and grade retention slightly, but they remain significant predictors for future dropout. The effect of gender on dropout is revealed in several studies and replicated in this study: boys have a significant higher chance for dropout, compared to girls. With an odds-ratio of 0.60 , girls have $40 \%$ lower odds to dropout, compared to boys. COGN has a significant effect on dropout $\left(p_{i} .001\right)$, whereas a one standard deviation increase in initial cognition $(\mathrm{SD}=0.62075)$, lowers the odds for dropout with $38 \%$. A one standard deviation increase in SES ( $\mathrm{SD}=2.17)$, lowers the odds for dropout with $47 \%$. The addition of these student characteristics already explains a substantial part of the differences between schools and we can state that the chance for dropout is strongly defined by intake characteristics and grade retention in secondary education, even before incorporating school characteristics. In 
an attempt to explain differences between schools in dropout rates, we also add school characteristics to our model. Four school characteristics are selected for our model. The best fit is obtained by adding the SES-S and RELAT-S variables. Including the COGN-S and GEN-S results in higher DIC-values and therefore, these variables are omitted from the final model. As can be found in Table 4, SES-S $\left(p_{i} .001\right)$ and RELAT-S $(p=0.017)$ have an effect on future dropout. Schools with higher mean socioeconomic composition, have far lower dropout rates. An increase of one standard deviation $(\mathrm{SD}=0.46152)$ in the mean socioeconomic composition of the school, lowers the chance to dropout in that school with approximately $44.4 \%$, even after controlling for student SES and other background characteristics. An increase of one standard deviation $(\mathrm{SD}=0.15327)$ in RELAT-S, reduces the risk to dropout in that school with $21 \%$. With the addition of these two school level variables, nearly all the between school variance can be explained.

\subsection{Assumptions of discrete-time hazard models}

Before drawing conclusions on the final model, we verify three important assumptions of the discrete-time hazard model: the proportionality assumption, the linearity assumption and the no unobserved heterogeneity assumption. Testing the proportionality assumption, one tests whether each predictor in the model has an identical effect in every time period under study. This is a very strong assumption and Singer and Willett (1991) argue that "violations of the proportionality assumption are the rule, not the exception' (p. 279). This assumption can be assessed by implementing interaction terms between the predictor and the time-variables (Grade). In our study, every interaction between the time variables and predictors was considered, but resulted in increasing DIC-values (not reported), indicating poorer model fits. Therefore, there is no reason to believe that the proportionality assumption has been violated. The linear additivity assumption, similar to the linearity assumption in linear regression, implies that a predictor's effect does not depend on the value of another predictor in the model. This assumption can easily be tested by looking for interactions between substantive predictors, and comparing the resulting model fits. In this study, only the SES-variable interacted significantly with both indicators of grade retention (RET_P and RET_S). Both interactions indicate a decreasing probability of dropping out for students with higher SES. However, this decrease is smaller when students are over-aged or retained in grade during secondary education. Both interactions are included in Model 10 and presented in Figure 1 and Figure 2.

The assumption of no unobserved heterogeneity, meaning that one or more important predictors have been omitted from the model, is the most difficult to assess. For checking this assumption, Singer and Willett (2003) recommend focusing on the hazard function, since unobserved heterogeneity has a consistent effect on the time variables and will lead to decreasing hazard functions. In the present study, the hazard functions increase over time (with a slight decrease in Grade 12), from which we can conclude that unobserved heterogeneity seems unproblematic. Effect of cross-classification on the final model Analogous to the comparison of the different baseline models, we compare estimates and variance components of the cross-classified approach with those of the last school approach. Additionally, we 
Fig. 1 Interaction-effect SESRET_P

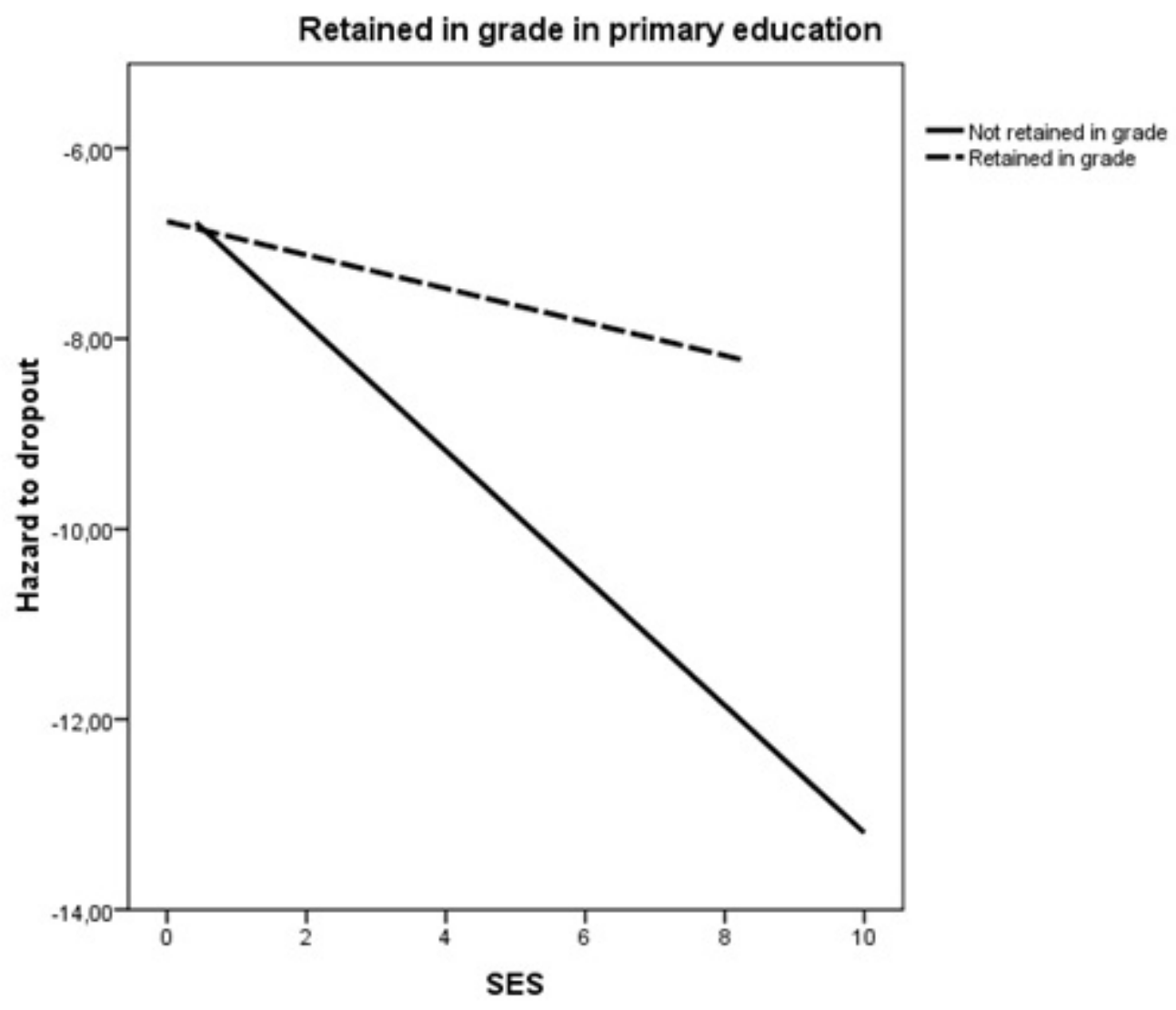

focus on the effect of ignoring the cross-classification on the standard errors of the estimates.

Comparing the last school approach with the model with cross-classifications, differences between these two models on the standard errors arise, similar to those in Luo and Kwok (2009). Compared to the cross-classified approach (Model 12), the standard errors associated with the time variables of the last school approach (Model 11) are substantially higher. In contrast, the standard errors associated with the school characteristics in the last school approach are lower, while the standard errors associated with the student characteristics remain unaffected. Following Luo and Kwok (2009), this was somewhat expected because ignoring a cross-classification results in an overestimation of the standard errors at the $(\mathrm{k}-$ 1)th level and an underestimation of the standard errors of the estimates of the variables related with the ignored crossed factor (at the $\mathrm{k}$-th level). The standard errors associated with the predictor variables of the remaining crossed factor at the k-th level, are found to remain stable. Applied to our last school approach, we ignore the fact that grades at Level-1 are crossed with students and schools 
Fig. 2 Interaction-effect SESRET_S

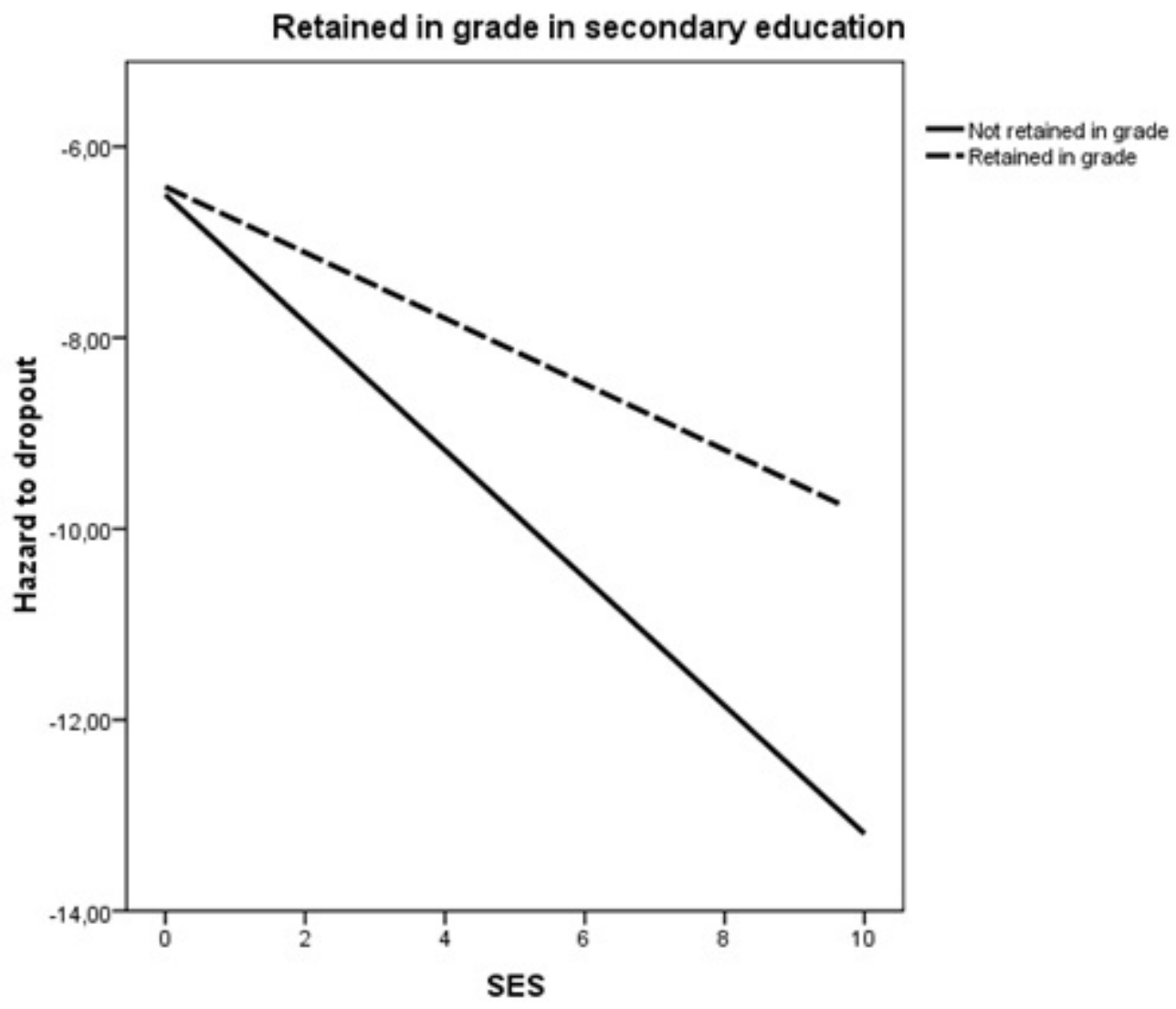

at Level-2. Ignoring this crossing at Level-2, resulted in higher standard errors of the regression coefficients of the predictor variables on Level-1 (Grade) and lower standard errors of the variables related with the ignored crossed factor (Schools). The standard errors associated with the student characteristics remain unaffected. The underestimation of standard errors can become problematic in terms of significance of the associated predictor, and can therefore lead to different conclusions. The variance components of our final model also differ depending on the classification. Ignoring the cross-classification affects the school level variance in a similar way as for the standard errors: the between school variance component in the last school approach seems to be an underestimation of the actual between school variance. Comparing the effects of school characteristics on the last school model and the cross-classified model emphasizes the importance of incorporating student mobility in dropout research. In the last school approach, a school with a socioeconomic composition (SES-S) one standard deviation $(\mathrm{SD}=0.46152)$ above the mean, reduces the probability to dropout in that school by $39 \%$, which is a smaller impact compared to the cross-classified approach. However, the ef- 
Table 5 impact of school characteristics in last school and cross-classification approach

\begin{tabular}{|c|c|c|c|c|}
\hline & \multicolumn{2}{|c|}{$\begin{array}{c}\text { Model } 11 \\
\text { Last school }\end{array}$} & \multicolumn{2}{|c|}{$\begin{array}{c}\text { Model } 12 \\
\text { Cross-classification }\end{array}$} \\
\hline & Est. & S.E & Est. & S.E \\
\hline Constant & -9.41 & 0.67 & -9.309 & 0.64 \\
\hline Grade 8 & $3.212^{* * *}$ & 0.661 & $3.110^{* * *}$ & 0.631 \\
\hline Grade 9 & $3.766^{* * *}$ & 0.656 & $3.600 * * *$ & 0.626 \\
\hline Grade 10 & $4.092^{* * *}$ & 0.655 & $3.912^{* * *}$ & 0.624 \\
\hline Grade 11 & $4.268 * * *$ & 0.655 & $4.078^{* * *}$ & 0.624 \\
\hline Grade 12 & $3.514^{* * *}$ & 0.663 & $3.317^{* * *}$ & 0.632 \\
\hline $\mathrm{RET}_{S}$ & $1.416^{* * *}$ & 0.155 & $1.418^{* * *}$ & 0.155 \\
\hline $\mathrm{RET}_{P}$ & $1.772^{* * *}$ & 0.148 & $1.766^{* * *}$ & 0.148 \\
\hline GEN & $-0.445^{* * *}$ & 0.11 & $-0.440 * * *$ & 0.11 \\
\hline COGN & $-0.689 * * *$ & 0.08 & $-0.669 * * *$ & 0.08 \\
\hline SES & $-0.677 * * *$ & 0.068 & $-0.670 * * *$ & 0.068 \\
\hline SES-S & $-1.101^{* * *}$ & 0.275 & $-1.269 * * *$ & 0.283 \\
\hline RELAT-S & -1.14 & 0.582 & $-1.419^{*}$ & 0.604 \\
\hline $\mathrm{RET}_{P} * S E S$ & $0.490 * * *$ & 0.068 & $0.494^{* * *}$ & 0.068 \\
\hline $\operatorname{RET}_{S} * S E S$ & $0.316^{* * *}$ & 0.071 & $0.325^{* * *}$ & 0.071 \\
\hline Between schools & 0.125 & 0.062 & 0.143 & 0.065 \\
\hline DIC & 3904 & & 388 & \\
\hline $\mathrm{N}$ & 285 & & 285 & \\
\hline
\end{tabular}

fect of perceived relationships at school (RELAT-S) indicates the importance of including student mobility. In the last school approach, this RELAT-S becomes non-significant, whereas in the cross-classified approach, RELAT-S is a significant predictor of future dropout. Besides the bias in standard errors and the nonsignificance of school level variables, also the lower DIC model fit indicates the superiority of the cross-classified model.

\section{Discussion}

Research on dropout in secondary education has been the topic of many studies and remains a contemporary topic. The interest for this dropout phenomenon is obvious: not only do dropouts have lower job opportunities (Solga 2002), when they do find a job, these jobs are characterized by a lower social status and lower earnings (Verhofstadt et al. 2007). These strong implications justify the permanent attention of researchers for dropout. However, past research has some methodological shortcomings. In this study, we attempted to address some of these shortcomings by adopting a multilevel discrete time hazard model. Although the hazard approach is the most appropriate method in studying dropout and was implemented in some dropout-research (e.g. Bowers 2010; Gesthuizen et al. 2005; Roderick 1994), past research which adopted this method ignored the hierarchical structure of educational data. When a multilevel approach was implemented, researchers did not correct for student mobility despite the strong association with student dropout. This discussion will focus on the two main topics of this study. At first, we discuss the results of the different approaches in modelling dropout and student mobility. The second part gives an overview of the results regarding 
the student and school characteristics predicting dropout in secondary education. Traditionally, past research on dropout, which implemented a hazard analysis, ignored the fact that students are nested within schools. Replicating this approach, and comparing this approach with multilevel approaches, this study shows an overestimation of the time variables and a higher DIC-value indicating a poorer fit. In a next step, we classify students on different school ID's, from which different results arise. In line with Grady and Beretvas (2010), models which are based on the school ID of the first school attended, yield the highest model fits and lowest between-school variance, indicating that this approach fails to explain some part of the variation in the data and should be avoided in future research. Their study, however, was limited in the fact that they did not focus on the last school approach. For dropout research, taking the ID of the school at which the student dropped out, seems more rational compared to the first school approach. Results of the last school approach indicate that, for this dataset, classifying students on the last school attended produces better fits and results in substantially higher between-school variance. Extending these approaches and taking into account the student mobility by means of a cross-classification with measurement occasions nested within a crossing of students and schools at Level-2, results in a better fit compared to the last school approach, which underlines the importance of incorporating the mobility. If we accounted for the student mobility by means of a multiple membership model, this resulted in a higher DIC-value compared to the last school approach, which was somewhat unexpected based on previous research. The poorer model fit stresses the importance of the choice of correct weighting schemes. In this study, we use the proportional time spent in every school, which seems, compared to the model fits of the cross-classified and last school approach, an oversimplification of the school effects on dropout. It seems to be the case that not every school attended has the same impact, and comparing the model fits of the last school, cross-classification and multiple membership approach indicates that more recent schools have a greater impact, as suggested by Fielding and Goldstein (2006). As mentioned, not only the time spent in a particular school is of importance, but also the timing and order the student was in different schools. By way of illustration (and because of common practice), we also estimate a model where we delete school changers. This deletion results in a reduction of our dataset of about $30 \%$ of the subjects, which makes a comparison with the previous approaches impossible. After the addition of student and school characteristics to the model, the impact of incorporating student mobility becomes more pronounced. We added these student and school characteristics to the crossclassified model and compared this model with the last school approach, since these two approaches yield the best model fits. In line with Luo and Kwok (2009), the standard errors associated with the time variables in the last school approach seem to be overestimated, in contrast to an underestimation of the standard errors of the parameters associated with the ignored crossed factor. More important is the impact on the fixed effects parameters. Where parameters associated with student characteristics remain relatively stable, parameters associated with school characteristics and the time variables change to a large(r) extent. Results indicate a similar underestimation of school characteristics for the last school approach, which led to insignificant school characteristics compared with the cross-classified approach. In general, these school level variables seem to have a stronger impact on the risk to dropout than generally assumed. This difference emphasizes the 
importance of adopting a cross-classified approach in studying dropout. The conclusions regarding dropout in secondary education are based on the cross-classified approach. The highest chance to dropout is in Grade 11, with a decrease in Grade 12. The highest chance in Grade 11 is not surprising since a lot of students who dropout were retained in grade and compulsory education ends at the age of 18 . Student characteristics predicting dropout are very similar to previous research (Alexander et al. 2001; Lee and Burkham 2003; Marks 2007). Our analyses confirm the negative impact of grade retention on dropout, as found in previous studies. Results indicate that grade retention is a strong indicator of future dropout, irrespective of the fact the student was retained in grade in primary or secondary education. This impact of grade retention remains, even after controlling for other relevant student background characteristics. In line with Alexander et al. (2001), we conclude that early achievement can also predict future dropout, even after correcting for grade retention in primary and secondary education. As for the effect of gender, our results are in line with e.g. Marks (2007): boys tend to have higher odds of leaving school without a diploma, compared to girls. The effect of the final student characteristic, socioeconomic status, was in line with previous research and highlights the impact of SES on dropout. Children stemming from a lower socioeconomic class, tend to have higher chances to dropout irrespective of their achievement. The final model in this study also presents an interaction between SES and the two variables related to grade retention, where the effect of SES on future dropout is different depending on the fact the student was retained in grade or not. Students retained in grade during primary education, tend to have high chances to dropout, regardless their SES-status. On the other hand, students who started secondary education on time, but are from a low SES-family, have a very high chance to dropout, compared to students from high SES-families. If a student was retained in grade during secondary education, the SES-status of the student will determine if a student drops out. Retained students with a high SES tend to have lower chances to dropout compared to retained students with a low SES. Regarding the effects of schools on dropout, both the socioeconomic composition and the relationships at school seem to predict the chances to dropout. In line with Rumberger and Thomas (2000), we conclude that high-SES schools have lower dropout rates compared to schools with an average SES-composition. We can draw similar conclusions regarding the effect of school climate on the chance to dropout: schools where the relationships between students and teachers are better, tend to have lower dropout rates.

\section{Conclusion}

With this study, we attempt to come towards some methodological shortcomings in previous dropout research. We implement a multilevel discrete-time hazard model, in which we correct for school changes by adopting a multiple membership approach and a cross-classified approach. These approaches are demonstrated on a real dataset. We conclude that ignoring the hierarchical nature of educational data, and ignoring student mobility can lead to different conclusions on the predictors of dropout. With this discrete-time approach, we are also able to draw conclusions on the moment of dropout and the implementation of time-varying variables be- 
comes straightforward. Overall, this study contributes to the modelling of dropout, accounting for student mobility.

\section{References}

Alexander, K., Entwisle, D., Kabbani, N.: The dropout process in life course perspective: Early risk factors at home and school. Teach. Coll. Rec. 103, 760-822 (2001)

Allison, P.: Discrete-time methods for the analysis of event histories. Sociol. Methodol. 13, 61-98 (1982)

Barber, J. S., Murphy, S. A., Axinn, W. G., Maples, J.: Discrete-time multilevel hazard analysis. Sociol. Methodol. 30, 201-235 (2000)

Bowers, A.: Grades and Graduation: A Longitudinal Risk Perspective to Identify Student Dropouts. J. Educ. Res. 103, 191-207 (2010)

Browne, W. J., Goldstein, H., Rasbash, J.: Multiple membership multiple classification (MMMC) models. Stat. Model. 1, 103-124 (2001)

Croninger, R.G., Lee, V.E.: Social capital and dropping out of high school: Benefits to at-risk students of teachers' support and guidance. Teach. Coll. Rec. 103, 548-581 (2001)

De Fraine, B.: Cognitieve en niet-cognitieve e?ecten van prestatiegericht klimaat, gemeen-schapsgericht klimaat en groepssamenstelling in klassen en scholen. Verkenning met het multiniveaumodel (Cognitive and non-cognitive effects of academic climate, communitarian climate and group composition of classes and schools. Explorations with multilevel models). Dissertation, K.U.Leuven, Belgium (2003) Finn, J.: Withdrawing from school. Rev. Educ. Res. 59, 117-142 (1989) Fielding, A.: Teaching Groups as Foci for Evaluating Performance in Cost-Effectiveness of GCE Advanced Level Provision: Some Practical Methodological Innovations. Sch. Eff. Sch. Improv. 13, 225-246 (2002)

Fielding, A., Goldstein, H.: Cross-classified and multiple membership structures in multilevel models: an introduction and review. University of Birmingham: Department for University Skills (2006)

Fredricks, J. A., Blumenfeld, P. C., Paris, A. H.: School Engagement: Potential of the Concept, State of the Evidence. Rev. Educ. Res. 74, 59-109 (2004)

Gesthuizen, M., De Graaf, P.M., Kraaykamp, G.: The changing family background of the low-educated in the Netherlands, socio-economic, cultural, and sociodemographic resources. European Sociol. Rev. 21, 441-452 (2005)

Goldschmidt, P., Wang, J.: When can schools affect dropout behavior? A longitudinal multilevel analysis. Am. Educ. Res. J. 36, 715-738 (1999)

Goldstein, H.: Methods in school effectiveness research. Sch. Eff. Sch. Improv. 8, 369-395 (1997)

Goldstein, H.: Multilevel statistical models. Arnold Publishers, London (2011)

Goldstein, H., Burgess, S., McConnell, B.: Modelling the e?ect of pupil mobility on school di?erences in educational achievement. J. Roy. Statistical Society 170, 941-954 (2007)

Goldstein, H., Sammons, P.: The Influence of Secondary and Junior Schools on Sixteen Year Examination Performance: A Cross-classified Multilevel Analysis. Sch. Eff. Sch. Improv. 8, 219-230 (1997)

Grady, M. W., Beretvas, S. N.: Incorporating student mobility in achievement 
growth modeling: A cross-classi?ed multiple membership growth curve model. Multivar. Behav. Res. 45, 393-419 (2010)

Hickman, G., Bartholomew, M., Mathwig, J., Heinrich, R.: Di?erential developmental pathways of high school dropouts and graduates. J. Educ. Res. 102, 3-14 (2008)

Jimerson, S., Anderson, G., Whipple, A.: Winning the battle and losing the war: Examining the relation between grade retention and dropping out of high school. Psychol. Schools 39, 441-457 (2002)

Kleinbaum, D. G., Klein, M.: Survival analysis. A Self-Learning Text. Springer, New York (2005)

Leckie, G.: The complexity of school and neighbourhood e?ects and movements of pupils on school differences in models of educational achievement. J. Roy. Stat. Soc. A Sta. 172, 537-554 (2009)

Lee, V., Burkam, D.: Dropping out of high school: The role of school organization and structure. Am. Educ. Res. J. 40, 353-393 (2003)

Luo, W., Kwok, 0.: The impacts of ignoring a crossed factor in analyzing crossclassified data. Multivar. Behav. Res. 44, 182-212 (2009)

Luyten, H., Bosker, R., Dekkers, H., Derks, A.: Dropout in the lower tracks of dutch secondary education: predictor variables and variation among schools. Sch Eff. Sch. Improv. 14, 373-411 (2003)

Marks, G.: Do schools matter for early school leaving? Individual and school in?uences in Australia. Sch Eff. Sch. Improv. 18, 429-450 (2007)

Opdenakker, M.C., Van Damme, J.: Differences Between Secondary Schools: study about school context, group composition, school practice, and school effects with special attention to public and Catholic schools and types of schools. Sch Eff. Sch. Improv. 17, 87-117 (2005)

Pampel, F.C.: Logistic Regression: A Primer. Sage University Papers Series on Quantitative Applications in the Social Sciences, 07-132. Sage, Thousand Oaks, CA. (2000)

Pustjens, H., Van de gaer, E., Van Damme, J., Onghena, P.: Curriculum choice and success in the first two grades of secondary education: students, classes, or schools? Sch. Eff. Sch. Improv. 19, 155-182 (2008)

Rasbash, J., Steele, F., Browne, W., Goldstein, H.: A user's guide to mlwin, v2.10 [Computer software manual]. University of Bristol (2009)

Raudenbush, S.W.: A Crossed Random Effects Model for Unbalanced Data With Applications in Cross-Sectional and Longitudinal Research. J. Educ. Stat. 18, 321349 (1993)

Raudenbush, S. W. Bryk, A. S.: Hierarchical Linear Models: Applications and Data Analysis Methods. Sage Publications, Thousand Oaks (2002)

Ream, R.K., Rumberger, R.W.: Student Engagement, Peer Social Capital, and School Dropout Among Mexican American and Non-Latino White Students. Sociol. Educ. 81, 109-139 (2008)

Roderick, M.: Grade retention and school dropout: Investigating the association. Am. Educ. Res. J. 31, 729-759 (1994)

Rumberger, R.: Dropping out of middle school: A multilevel analysis of students and schools. Am. Educ. Res. J. 32, 583-625 (1995)

Rumberger, R.: Why students drop out of school and what can be done. In Dropouts in America: How severe is the problem? What do we know about intervention and prevention? Cambridge, MA: Harvard University (2001) 
Rumberger, R., Thomas, S.: The distribution of dropout and turnover rates among urban and suburban high schools. Soc. Educ. 73, 39-67 (2000)

Singer, J., Willett, J.: Modeling the days of our lives: Using survival analysis when designing and analyzing longitudinal studies of duration and the timing of events. Psychol. Bull. 110, 268-290 (1991)

Singer, J., Willett, J.: Applied longitudinal data analysis: Modeling change and event occurrence. Oxford University Press, Oxford, USA (2003)

Snijders, T., Bosker, R.: Multilevel Analysis. An introduction to basic and advanced multilevel modeling. Sage Publications, London (1999)

Solga, H.: Stigmatization by negative selection. Explaining less-educated people's decreasing employment opportunities. Eur. Sociol. Rev. 18, 159-178 (2002)

South, S., Haynie, D., Bose, S.: Student mobility and school dropout. Soc. Sci. Res. 36, 68-94 (2007)

Spiegelhalter, D., Best, N., Carlin, B., van der Linde, A.: Bayesian measures of model complexity and fit. J. Roy. Stat. Soc. B Met. 64, 583-639 (2002)

Teitler, J.O., Weiss, C.C.: Effects of neighborhood and school environments of transitions to first sexual intercourse. Soc. Educ. 73, 112-132 (2000)

Van Damme, J., De Fraine, B., Van Landeghem, G., Opdenakker, M. C., Onghena, P.: A new study on educational effectiveness in secondary schools in Flanders: An introduction. Sch. Eff. Sch. Improv. 13, 383-397 (2002)

Van Damme, J., De Troy, A., Meyer, J., Minnaert, A., Lorent, G., Opdenakker, M.C., Verduyckt, P.: Succesvol doorstromen in de aanvangsjaren van het secundair onderwijs (Successful passing through the first years in secondary education). Acco, Leuven (1997)

Verhofstadt, E., De Witte, H., Omey, E.: Higher educated workers: better jobs but less satisfied? Int. J. Manpower 28, 135-151 (2007) 